\title{
L'enseignement supérieur britannique entre deux feux
}

Great Britain: Higher Education in question

La enseñanza superior británica entre dos fuegos

\section{Muriel Robinson}

Traducteur : Isabelle Richard

\section{OpenEdition}

\section{Journals}

Édition électronique

URL : http://journals.openedition.org/ries/2537

DOI : $10.4000 /$ ries. 2537

ISSN : 2261-4265

Éditeur

Centre international d'études pédagogiques

\section{Édition imprimée}

Date de publication : 1 juin 2000

Pagination : $97-108$

ISSN : 1254-4590

\section{Référence électronique}

Muriel Robinson, «L'enseignement supérieur britannique entre deux feux », Revue internationale d'éducation de Sèvres [En ligne], 26 I juin 2000, mis en ligne le 01 juin 2003, consulté le 02 mai 2019. URL : http://journals.openedition.org/ries/2537 ; DOI : 10.4000/ries.2537 


\section{L'enseigement supérieur britannique entre deux feux ${ }^{1}$}

Muriel Robinson ${ }^{2}$

\section{Résumé}

Compte tenu des modes de financement actuels de l'enseignement en Angleterre, les départements d'enseignement supérieur sont évalués et financés par deux agences respectives, dont l'une - fondée en grande partie sur de l'autoévaluation - semble fonctionner de manière plus rigoureuse et satisfaisante.

\section{Great Britain: Higher Education in question}

Models for higher education in England have led to a situation where aspects of work within education departments may be funded and inspected by two different government agencies. The approaches of these two agencies are compared and the case made that one of these, which involves significant self-evaluation, is a more robust and worthwhile model.

\section{La enseñanza superior británica entre dos fuegos}

Debido a los modos de financiación actuales de la enseñanza en Inglaterra, dos agencias evalúan y financian los departamentos de enseñanza superior. La agencia, que se basa en gran parte sobre la autoevaluación, parece funcionar más rigurosa y satisfactoriamente.

1 Cet article a été traduit par Isabelle Richard.

2 Les opinions exprimées dans cet article sont celles de l'auteur et n'engagent en rien les établissements mentionnés. 
La complexité des systèmes de financement de l'enseignement supérieur britannique, sans doute bien peu cohérents aux yeux des étudiants, fait que la formation est financée et, pour ce qui nous concerne plus directement dans cet article, évaluée par deux organes distincts. L'ensemble du travail réalisé avec les personnes dotées du qualified teacher status ${ }^{3}$ est financé par la Teacher Training Agency (TTA). Les autres étudiants, impliqués dans l'enseignement post-scolaire ou pour adultes, ainsi que les enseignants universitaires, le personnel de santé, les travailleurs sociaux, les professeurs-assistants et les aides maternelles du préscolaire, sont rémunérés par le Higher Education Funding Council for England (HEFCE).

En conséquence de cette dichotomie, les travaux financés par la TTA sont évalués par l'organisme gouvernemental en charge de l'inspection des écoles et de la supervision de la formation initiale et continue des enseignants, l'Office for Standards in Education (OFSTED). Les travaux financés par l'HEFCE font eux l'objet

Sans aucune implication de sa part, la profession se sent désarmée et suspicieuse à l'égard de la validité de normes d'évaluation qui ont été émises sans faire référence au savoir ni à la qualification des professionnels

d'une évaluation réalisée par la Quality Assurance Agency for Higher Education (QAA). Contrairement à l'OFSTED qui qualifie sa procédure évaluative d' "inspection ", la QAA parle d' "audit " ou dans ce cas précis, d' " audit par matière ». Cette variante terminologique reflète une différence idéologique qui est ressortie très clairement par la suite, lors des visites des deux organismes, entre janvier 1999 et février 2001. Cet article compare les deux approches et cherche à déterminer laquelle des deux est la plus rigoureuse en matière d'auto-évaluation et d'amélioration personnelle.

\section{Le couple financement et évaluation}

Avant d'étudier les deux systèmes plus en détail, il est important d'en préciser les implications pour une institution d'enseignement supérieur. À Brighton, par exemple, la School of Education a instauré avec succès un programme de formation professionnelle continue intégré à un système de notation par module, applicable à tous les étudiants, depuis les professeurs-assistants qui font leurs premiers pas dans l'enseignement supérieur jusqu'aux étudiants en fin de cursus. Ce système de notation, destiné à garantir progression et continuité à l'ensemble des étudiants, inclut des procédures normalisées d'admission, de stage, d'évaluation et de contrôle de qualité.

3 Diplôme d'habilitation à l'enseignement. 
La formation continue des professeurs y est dispensée grâce à des fonds provenant de trois sources différentes de la TTA. On dispose ainsi des fonds de transition résiduels ( $75 \%$ du budget en 1998-1999 et $50 \%$ en 2000), des fonds émanant d'une première série de subventions - destinés à une large gamme de modules - et des fonds liés à d'autres subventions, d'un montant plus réduit. Seules les prestations dépendant de la première série de subventions ont fait l'objet d'une évaluation et parmi les huit domaines qu'elles recouvrent, trois seulement ont été réellement contrôlés qui ne constituent qu'un faible échantillon des prestations totales financées par la TTA (10\% environ du nombre total d'étudiants). Ce contrôle s'est déroulé de janvier 1999 à mars 2000 ; les projets de rapports et de notes ont été envoyés dès juillet 2000. Les autres programmes de formation continue, financés par le HEFCE, font partie des études liées à l'enseignement et doivent en ce sens se soumettre à un audit par matière se déroulant en fin de processus, entre septembre 2000 et décembre 2001.

Cet audit tiendra compte de la prestation dans son ensemble pour un domaine précis. La première visite a débuté en septembre 1999. La première étape, consistant en la préparation et la présentation du document d'auto-évaluation (self-assessment document, SAD), s'est achevée au début de juillet 2000. La proximité des visites respectives et la préparation simultanée aux contrôles des deux organismes ont révélé des contrastes détaillés plus loin. Ces derniers ont été plus particulièrement mis en relief par les récentes remarques dans la presse d'un inspecteur principal des écoles de sa Majesté ${ }^{4}$. Selon lui, il serait souhaitable que l'OFSTED disposât des mêmes attributions que la QAA en matière d'enseignement universitaire, afin de garantir un processus plus rigoureux du contrôle de la qualité de l'enseignement supérieur en Angleterre.

À première vue et sous de nombreux aspects, les deux modèles semblent similaires. Je souhaiterais, tout d'abord, mettre en lumière ces ressemblances assez brièvement avant de m'attacher à la différence majeure et de montrer quelle procédure semblerait plus apte à apporter des changements dans le système. La publication des objectifs de chaque agence montre de nombreuses similitudes. Le site web de l'OFSTED décrit ainsi ses attributions : «Améliorer les normes de niveau et de qualité de l'enseignement par le biais d'une inspection indépendante et régulière, de la publication de rapports et de recommandations autonomes et éclairées $»^{5}$. Ces attributions impliquent donc l'inspection des écoles et des programmes d'enseignement initiaux destinés aux professeurs (teacher training programmes, ITT). En effet, chaque année l'OFSTED visite les Schools of Education afin d'inspecter plusieurs programmes ITT. Toutefois, dans le cadre d'une formation continue bénéficiant de subventions, l'accent est mis sur la façon dont la prestation correspond aux critères d'allocation des fonds.

4 Woodhead Cris, "Outsiders Looking ", in Times Higher Education Supplement, 10 mars 2000.

5 http://www.ofsted.gov.uk/about/intro.htm 
Ces critères se fondent sur le principe selon lequel seule une qualité accrue de la formation continue pourra aider les professeurs à élever le niveau des élèves, à relever les défis nouveaux tout en amenant les résultats globaux de l'école à se soumettre aux procédures appropriées de garantie du niveau de qualité ${ }^{6}$.

Selon le site web de la QAA, les objectifs de l'évaluation de la qualité sont les suivants :

- s'assurer que les fonds publics financent un enseignement d'une qualité acceptable,

- diffuser des informations sur cet enseignement par le biais de la publication de rapports,

- fournir des informations et des avis incitant à une amélioration de l'enseignement ${ }^{7}$.

Remarquons ici l'importance accordée aux fonds publics, qui ne sont pas explicitement mentionnés par l'OFSTED. Les deux organismes insistent tous deux sur la publication des rapports et la diffusion d'une bonne formation. En d'autres termes, l'objectif est dans chaque cas une prestation de haute qualité dispensée par des prestataires de services variés.

Les critères établis comme cadre de référence de chaque agence semblent appréhender différemment la prestation, mais les similitudes demeurent incontestables. Les critères de l'OFSTED sont l'identification des besoins, la prestation, l'impact et la garantie de la qualité. Il attribue une note pour chaque domaine séparément.

L'audit par matière de la QAA consiste à observer la prestation selon six domaines :

- conception des curricula,

- contenu et organisation,

- enseignement, apprentissage et évaluation,

- progression et niveau des étudiants,

- soutien et suivi des étudiants,

- ressources et enseignement,

- gestion et amélioration de la qualité.

Chaque domaine est noté séparément, évitant toute confusion, bien que ce soit monnaie courante dans les médias et dans les établissements. La terminologie est, certes, différente mais les problèmes soulevés par l'OFSTED sont présents dans les considérations de la QAA et inversement.

Les deux agences publient des documents décrivant plus en détail la procédure d'inspection/audit. Il est important de rappeler à ce stade que l'OFSTED n'en est qu'à sa première inspection de la formation continue, alors que l'audit par matière de la QAA est presque terminé pour ce qui est du second

6 OFSTED, 1999, Annexe A., Chapitre A.

7 http://www.niss.ac.uk/education/qaa/subjectreviews/subjectreviews $2 \mathrm{htm}$ 
cycle des visites de garantie du niveau de qualité en milieu universitaire, bien qu'il s'agisse du premier audit d'un cursus formant à l'enseignement. Il n'est donc pas surprenant que les informations de la QAA, disponibles plus tôt, soient plus complètes ( 51 pages contre 28 ) et qu'elles revêtent un caractère plus professionnel. Les deux agences suivent des procédures qui, à première vue, pourraient sembler identiques, comportant la même tactique de sollicitation de documents préliminaires, de visite au prestataire de services, d'observation de l'enseignement, de recueil d'informations auprès du personnel enseignant et de direction, des étudiants actuels et anciens et des autres partenaires.

À la fin de la procédure, les deux publications sont d'abord communiquées aux établissements qui vérifient leur exactitude. Les deux procédures sont déterminantes pour les financements à venir. La nouvelle série de subventions destinées à la formation continue requiert des informations précises sur les actions effectivement entreprises par les prestataires de services à la lumière des résultats de l'inspection, à la fois en termes spécifiques et, de manière plus générale, en relation avec les points forts et les points faibles des prestations inspectées par I'OFSTED. Il n'y a certes pas encore de relation directe entre une mauvaise évaluation de l'OFSTED et une éventuelle baisse du nombre d'étudiants dans les établissements de formation initiale des enseignants, bien qu'implicitement, la procédure d'offre n'écarte pas complètement cette éventualité. De même, un mauvais résultat attribué par la QAA peut conduire les établissements à perdre leur accréditation d'enseignement pour certaines matières.

\section{Entre inspection et audit : des différences philosophiques}

Les lecteurs pourront se demander pourquoi, si les procédures sont à ce point similaires et les conséquences d'importance égale, l'implication dans l'une ou l'autre des deux procédures soulève tant de difficultés. Voyons à présent la différence majeure existant entre les deux systèmes et comment leurs disparités, apparemment mineures, reflètent également une différence fondamentale de philosophie qui n'apparaît pas dans les objectifs cités par les deux agences, tels qu'ils ont été décrits ci-dessus. L'OFSTED réalise une inspection fondée sur un ensemble d'exigences qui ne prennent pas en compte les points de vue particuliers du prestataire de services et qui sont également utilisées afin de limiter le financement de la TTA. Les écoles et les établissements de formation continue sont supposés dispenser les cours en fonction des recommandations émises par la TTA. Il n'y a pas lieu ici de débattre sur l'enseignement à prodiguer. Mais en dépit des sessions consultatives régulières visant à donner l'apparence d'un engagement réel des professionnels, la plupart des personnes impliquées dans la formation des enseignants se sentent dépossédées du contrôle sur la formation initiale et 
continue. Le sens des responsabilités professionnelles est alors fort peu développé quand il s'agit de faire des choix éclairés, fondés sur un jugement indépendant. Un tel sentiment s'accroît quand l'inspecteur principal fait part de sa conviction selon laquelle seule une procédure d'inspection totalement indépendante est digne d'intérêt : "Le corps des inspecteurs doit être indépendant du gouvernement, d'une part, et de la profession, d'autre part, et ses conclusions doivent respecter des normes objectives et transparentes. ${ }^{8}$

Malheureusement, sans aucune implication de sa part, le corps enseignant se sent désarmé et suspicieux à l'égard de la validité de normes qui ont été émises sans faire référence ni au savoir, ni à la qualification des professionnels.

À l'inverse, l'approche de la QAA met l'accent sur un audit effectué par des pairs. L'évaluation des prestataires de services vise à déterminer dans quelle mesure la prestation correspond aux objectifs qu'ils se sont fixés. L'exercice initial d'auto-évaluation est fondamental dans la procédure, comme le montre l'énumération des étapes de l'audit :

- audit des pairs,

- auto-évaluation,

- comparaison des résultats obtenus et des objectifs,

- évaluation de la prestation,

- visite,

- notation de la prestation,

- décision générale/bilan, et rapport d'audit par matière. ${ }^{9}$

Ces éléments s'inscrivent dans un contexte de coopération : l'audit est en effet réalisé dans un esprit de dialogue et de collaboration entre l'établissement, le personnel en relation avec la matière enseignée, le médiateur de l'établissement et l'équipe d'audit. ${ }^{10}$

En insistant sur l'audit des pairs et les éléments d'auto-évaluation, la QAA présente une philosophie bien moins inquisitoriale et condescendante que celle de l'OFSTED, ce qui fait sa force.

\section{Les conséquences de l'évaluation sur le système}

Avant d'expliquer pourquoi le dialogue et la coopération, essentiels aux yeux de la QAA, ont plus d'impact sur l'enseignement que les conclusions émanant de l'OFSTED, rappelons que la différence est perceptible dans la procédure d'évaluation. Les inspections de l'OFSTED sont réalisées par les inspecteurs de sa Majesté (Her Majesty's Inspectors, HMIs) et par les inspecteurs auxiliaires

$8 \mathrm{lbid}$, note 4.

9 QAA, 2000, p 14.

$10 \mathrm{lbid}$, note 9. 
(Additional Inspectors, AIs). Les HMIs sont salariés permanents de l'OFSTED, le responsable d'une inspection étant toujours un HMI. Les AIs sont recrutés par voie de presse ; ils sont issus de divers horizons et formés par l'OFSTED. Cette tactique est quelque peu hasardeuse, dans la mesure où il peut y avoir un écart entre leur spécialisation et la matière inspectée, compte tenu de la charge de travail et de leur disponibilité. Ainsi, un nouvel AI participant récemment à l'inspection du programme de langue pour le primaire croyait que le mot anglais cat se composait de trois syllabes, révélant ainsi une connaissance pour le moins excentrique et douteuse de la langue anglaise ! En outre, il est évident qu'il n'y a qu'un point de vue unique porté sur chaque cours. L'évaluation est ainsi le fait d'une seule et même personne. Bien que la procédure soit en théorie conçue pour éliminer tout jugement biaisé, l'expérience montre que l'inspecteur émet seul un avis sur une matière enseignée, ce qui donne un poids démesuré à son jugement qui ne fait l'objet ni de vérification, ni d'évaluation externe.

La QAA met donc l'accent sur l'audit de pairs. L'équipe est ainsi constituée de pairs académiques et professionnels pour une matière donnée. Tous les établissements doivent proposer des candidats qui, après sélection, sont formés par la QAA en partenariat avec la Universities and Colleges Staff Development Agency (UCoSDA). La plupart d'entre eux travaillent dans l'enseignement supérieur, mais peuvent exercer une autre profession en tant que libéral, dans l'industrie, etc. Il est possible de consulter un registre des personnes en charge de l'audit par matière. La taille de l'équipe, qui compte au minimum trois personnes, varie en fonction de l'envergure des prestations à examiner. Des réunions sont organisées avant la visite, en guise de préparation, et durant le déroulement de celle-ci. L'équipe au grand complet est présente durant la totalité de la visite et les conclusions sont le fruit d'un travail collectif de ses membres, qui prennent chaque jour le soin de faire un rapport sur leurs observations personnelles. Même si le responsable de l'audit appartient à une discipline différente, il a bénéficié d'une formation en gestion d'équipe et suivi un cours de spécialisation dans la matière à évaluer.

\section{Évaluation globale ou individualisée?}

Une autre différence majeure est apparue dans la nature même de la visite. Lors des inspections de l'OFSTED, le nombre de cours ou modules à inspecter est déterminé de façon centralisée, en fonction du niveau de financement accordé. Six jours maximum, préparation et rédaction incluses, sont octroyés pour chaque cours. Deux visites sur le site sont prévues et généralement effectuées par deux équipes successives.

Concrètement, Brighton a reçu quatre visites d'une demi-journée chacune, en comptant la visite préliminaire, une visite de contrôle d'enseignement de l'inspecteur principal, ainsi que deux autres visites de même nature effectuées 
par un inspecteur auxiliaire. Une visite a été consacrée aux prestations fournies à l'extérieur. Ces quatre demi-journées se sont étalées sur une quinzaine de mois. Une fois sur place, les inspecteurs lisent la documentation, assistent à des cours (trois séances de deux heures), rapportent leurs observations au responsable de la discipline et discutent de la prestation avec les membres clés de l'équipe de direction. Les longs intervalles entre les visites ont donné une allure quelque peu décousue à la procédure, et les inspecteurs n'ont que rarement tenté de s'impliquer de façon plus globale dans l'enseignement, se concentrant seulement sur un très petit échantillon de cours désignés lors de la première réunion et ne prenant connaissance que d'une documentation très générale sur les modules concernés.

L'audit par matière de la QAA n'a pas encore eu lieu, mais suivra très certainement le schéma détaillé dans les instructions. Il se tiendra donc sur trois jours et demi, et se déroulera comme à l'accoutumée du lundi après-midi au jeudi après-midi. La date effective a été précisée environ un an auparavant afin de convenir à tous. Une fois la visite commencée, l'équipe d'audit dispose d'une salle réservée à son usage personnel où elle trouve toute la documentation relative à l'auto-évaluation. Elle peut être écrite, provenir du web ou de tout autre support audio-visuel. Le temps passé dans la salle est réservé à la lecture, aux discussions et aux comptes rendus effectués à la lumière des informations tirées de la visite. Un certain nombre de réunions sont organisées avec les groupes responsables de chacun des six aspects de la prestation, des étudiants anciens et actuels ayant suivi ou suivant les cours présentement évalués, mais aussi avec les employeurs et les différents partenaires. De même, des visites de locaux hors site et de collèges associés à la formation ainsi que la participation à des cours sont prévues. L'équipe d'audit choisit les cours auxquels elle souhaite assister à partir de l'emploi du temps général de la semaine concernée ; tous les professeurs inclus dans le planning doivent donc se tenir prêts à une éventuelle évaluation. Cette approche bien plus globale ne prend certes en compte qu'une seule semaine, mais elle permet d'évaluer un échantillon plus représentatif de la matière telle qu'elle est enseignée dans son ensemble. Cette approche donne une meilleure idée de la nature effective de la prestation.

\section{À la recherche du dialogue}

Une autre différence importante est la création, par la QAA - qui envisage son audit comme un dialogue - d'un poste de médiateur d'établissement : généralement un membre senior spécialisé dans les normes scolaires. Le médiateur maintient le lien entre les personnels en relation avec la matière enseignée et l'équipe chargée de l'audit, ce qui lui permet d'assister à toutes les réunions in situ, sauf celle de l'attribution des notes. Le médiateur qui est formé par la QAA, peut ainsi informer l'équipe sur tout problème éventuel nécessitant une clarification. Les quatre jours de visite constituent un tout et tiennent compte de 
toutes les observations émises pendant leur durée. Ainsi, l'objectif de l'audit est d'obtenir l'évaluation la plus globale possible sans chercher à prendre en défaut la prestation évaluée, ni traquer les éventuelles erreurs. Le médiateur est un élément déterminant qui rend le dialogue plus concret.

Signalons à présent une différence mineure, mais qui mérite d'être soulignée. Bien que l'OFSTED et la QAA utilisent tous deux un système de notation allant de 1 à 4 , 1 est la meilleure note pour l'OFSTED alors que c'est 4 pour la QAA (voir tableau 1).

\section{Tableau 1}

\section{Les indicateurs du système de notation}

\begin{tabular}{|l|l|}
\hline OFSTED $^{11}$ : & QAA $^{12}$ : \\
\hline $\begin{array}{l}\text { 1. Très bonne qualité ; résultats excellents sous } \\
\text { plusieurs aspects }\end{array}$ & $\begin{array}{l}\text { 1. Les objectifs fixés par l'établissement ne sont } \\
\text { pas atteints ; des lacunes majeures doivent } \\
\text { être comblées }\end{array}$ \\
\hline 2. Bonne qualité sans faiblesse particulière & $\begin{array}{l}\text { 2. Les objectifs fixés sont atteints d'une façon } \\
\text { acceptable, mais des améliorations impor- } \\
\text { tantes pourraient être réalisées ; les objectifs } \\
\text { sont atteints de façon trop imprécise }\end{array}$ \\
\hline $\begin{array}{l}\text { 3. Résultats corrects nécessitant toutefois des } \\
\text { améliorations non négligeables }\end{array}$ & $\begin{array}{l}\text { 3. Les objectifs fixés sont atteints de façon } \\
\text { concluante, mais des améliorations sont enco- } \\
\text { re possibles }\end{array}$ \\
\hline 4. Qualité insuffisante & 4. Les objectifs fixés sont pleinement atteints \\
\hline
\end{tabular}

Notons que les qualificatifs de la QAA ont une signification et des implications plus précises. Généralement, quand une matière obtient une note inférieure à 4, l'élément qui pose précisément un problème est identifié. Les centres de ressources pourront ainsi perdre un point si les étudiants n'utilisent pas activement les ressources fournies, quelle que soit leur qualité. Avec la méthode de la QAA, le capital de départ est de 4 pour chacun des aspects; la note la plus élevée est ainsi accessible et il est rare qu'un établissement n'obtienne aucun 4 pour une seule et même matière. Les rapports étant publiés rapidement, les problèmes rencontrés dans d'autres établissements ou pour d'autres matières

11 Office for Standards in Education, Inspection of TTA-Funded INSET: Inspection Procedures, février 2000, non publié mais utilisé dans toutes les inspections de l'OFSTED.

12 Quality Assurance Agency for Higher Education, Subject Review Handbook. Gloucester: Quality Assurance Agency for Higher Education, 2000. 
peuvent servir de base à d'éventuelles améliorations lors de la préparation de l'audit, comme il est précisé plus loin. Avec la méthode de l'OFSTED, de telles conditions ne sont pas explicites. L'OFSTED part ainsi du résultat le moins bon comme base d'évaluation. Une majorité, voire une totalité de 1 est inhabituelle de la part de cet organisme.

Le manque de précision des indicateurs et leur formulation rend plus ardue l'identification précise des faiblesses de la prestation. À la suite d'un projet de rapport reçu début juillet, la School of Education de Brighton a dû combler ses lacunes, début octobre, avant de soumettre ses demandes de subvention pour la série suivante de financement. Il ne s'agit certainement pas de la meilleure façon d'obtenir des améliorations durables.

L'évaluation faite par des pairs, la nature de la visite intensive et la clarté des jugements émis rendent la procédure et le système plus rigoureux. La préparation à l'audit de la QAA a eu un bien plus grand impact sur l'auto-évaluation que la préparation à l'inspection de l'OFSTED. Le document d'auto-évaluation joue un rôle primordial car il permet de mener une enquête très minutieuse sur toutes les procédures de formation et d'identifier les éléments nécessitant une amélioration afin d'entreprendre des actions avant l'audit. À l'inverse, l'OFSTED exige simplement la mise à disposition d'informations telles que des exemples de publicités, des manuels d'étudiants, les programmes dans leurs grandes lignes et les curriculum vitae des membres du personnel. Cette tâche peut être effectuée par une seule personne en quelques heures seulement, alors que la QAA demande une documentation générale concernant les objectifs des enseignants, rédigée en termes précis. On doit ensuite comparer ces derniers aux résultats obtenus pour chacun des six aspects, en un nombre de mots définis. La documentation ainsi fournie sert de toile de fond à la visite et doit paraître familière et identifiable aux yeux du personnel et des étudiants. Elle doit être évaluative plus que descriptive et dans la mesure du possible, savoir identifier les problèmes qui nécessitent une solution, tout en mettant en lumière les points forts. La conception de ladite documentation est donc le fruit d'un effort commun ; des groupes sont chargés de travailler sur chacun des aspects pendant six mois avant la date finale de remise du document. Ces groupes rassemblent des administrateurs et des membres du corps enseignant, et recueillent le témoignage des étudiants. Ils ont tout d'abord réalisé des analyses de chaque aspect à partir d'un certain nombre d'items (atouts, faiblesses, occasions, menaces), en les confrontant de manière critique aux objectifs fixés. Ces derniers ont également retenu l'attention de l'ensemble des groupes. Chaque membre du personnel impliqué de façon significative dans la prestation doit participer au moins à un groupe, y compris les membres du personnel à temps partiel. Chaque groupe est pourvu d'un coordinateur. Les réunions régulières entre coordinateurs ont permis de comparer leurs notes et de parer aux problèmes rencontrés dans les divers aspects étudiés. Ainsi s'opère une réelle prise de conscience des démarches urgentes à entreprendre en identifiant les lacunes des démarches de formation.

Par conséquent, au moment de l'élaboration du document administratif 
unique, un réel examen des pratiques a déjà été entrepris et certains progrès réalisés. Par exemple, on constate fréquemment des faiblesses en matière de communication avec les centres de ressources, les personnels de la bibliothèque et du service informatique n'étant pas toujours impliqués de façon adéquate dans les décisions. L'inclusion dans le groupe du responsable informatique occasionne une nette amélioration en termes de communication, en ouvrant un espace de dialogue. En outre, cette décision se révèle bénéfique pour les étudiants et permet une plus grande adéquation entre les prestations et les besoins. Il apparaît aussi nettement que, malgré une identification des sites d'études extérieurs et de l'enseignement qui s'y déroule, les outils documentaires de ces sites font parfois défaut et que leur organisation manque de cohérence. Un grand nombre d'actions sont ainsi envisagées, de sorte qu'au moment où le document administratif est rédigé, les enseignants sont beaucoup plus confiants face à la qualité de leurs prestations et à ce qui leur reste à accomplir.

Mais, toutes ces actions n'engagent pas toujours une contribution aussi importante que ces exemples peuvent le suggérer. La section du document la plus difficile à rédiger est celle de l'enseignement, de l'apprentissage et de l'évaluation, parce que cela touche à la fois à la nature de la tâche et aux moyens utilisés. Très souvent, au moment de rédiger cette partie, on réalise que l'on a jusqu'à présent opté pour une conception de ces processus, tacitement partagée, qui manque d'articulation. On considérait son propre travail ordinaire comme correct et on s'aperçoit que d'autres en font de même. Lorsqu'on s'efforce d'exprimer ses convictions les plus profondes en matière de pédagogie avec l'aide d'un médiateur, on réalise que cet effort d'expression est, à bien des égards, également la relation dialectique avec la pratique que l'on cherche à établir chez les étudiants avec leur propre travail. Cette rédaction du document final se révèle finalement très représentative de ce qui compte dans l'enseignement. Or, rien, à ce niveau du débat, n'est inspiré explicitement ou incidemment par l'OFSTED.

Encore reste-t-il à savoir comment la QAA évaluera le travail et ce que sera également la version finale du rapport de l'OFSTED et les notes afférentes. Quelle que soit l'issue de ces deux procédures d'évaluation, il est déjà clair que la procédure de dialogue de la QAA aura eu un impact favorable sur la formation et l'enseignement, et qu'elle aura beaucoup plus incité à mettre en place des améliorations fondamentales dans la pratique et l'articulation de cette pratique. La procédure d'auto-évaluation et l'approche globale ont été beaucoup plus élaborées et bénéfiques que l'inspection de l'OFSTED. L'inspecteur en chef prétend que «le grand avantage de l'OFSTED est son regard neuf posé sur chaque chose, qui défie l'immobilisme et bénéficie à l'établissement inspecté ${ }^{13}$. Nous pensons 
que les évaluations de la QAA effectuées par les pairs ont incité à tout considérer d'un oeil neuf, bien plus que les inspections. Espérons que, même si l'OFSTED a gagné une plus grande influence sur l'enseignement supérieur, le modèle de la QAA sera appliqué à toute la formation des enseignants.

13 lbid, note 4. 